\title{
Predicting the Suitability of Service Animals Using Instrumented Dog Toys
}

\author{
CEARA BYRNE, Georgia Institute of Technology \\ JAY ZUERNDORFER, Georgia Institute of Technology \\ LARRY FREIL, Georgia Institute of Technology \\ XIAOCHUANG HAN, Georgia Institute of Technology \\ ANDREW SIROLLY, Georgia Institute of Technology \\ SCOTT GILLILAND, Georgia Institute of Technology \\ THAD STARNER, Georgia Institute of Technology \\ MELODY JACKSON, Georgia Institute of Technology
}

\begin{abstract}
Working dogs ${ }^{1}$ are significantly beneficial to society; however, a substantial number of dogs are released from time consuming and expensive training programs because of unsuitability in behavior. Early prediction of successful service dog placement could save time, resources, and funding. Our research focus is to explore whether aspects of canine temperament can be detected from interactions with sensors, and to develop classifiers that correlate sensor data to predict the success (or failure) of assistance dogs in advanced training. In a 2-year longitudinal study, our team tested a cohort of dogs entering advanced training in the Canine Companions for Independence (CCI) Program with 2 instrumented dog toys: a silicone ball and a silicone tug sensor. We then create a logistic model tree classifier to predict service dog success using only 5 features derived from dog-toy interactions. During randomized 10-fold cross validation where 4 of the 40 dogs were kept in an independent test set for each fold, our classifier predicts the dogs' outcomes with $87.5 \%$ average accuracy. We assess the reliability of our model by performing the testing routine 10 times over 1.5 years for a single suitable working dog, which predicts that the dog would pass each time. We calculate the resource benefit of identifying dogs who will fail early in their training, and the value for a cohort of 40 dogs using our toys and our methods for prediction is over $\$ 70,000$. With CCI's 6 training centers, annual savings could be upwards of $\$ 5$ million per year.
\end{abstract}

CCS Concepts: $\cdot$ Human Centered Computing $\rightarrow$ Ubiquitous and mobile computing $\rightarrow$ Ubiquitous and mobile computing systems and tools

\section{KEYWORDS}

Tangibles, tangible computing, smart objects, service dogs, temperament.

\section{ACM Reference format:}

Ceara Byrne, Jay Zuerndorfer, Larry Freil, Xiaochuang Han, Andrew Sirolly, Scott Gilliland, Thad Starner, and Melody Jackson. Predicting the Suitability of Service Animals Using Instrumented Dog Toys. Proc. ACM Interact. Mob. Wearable Ubiquitous Technol. 1, 4, Article 127 (December 2017), 20 pages. https://doi.org/10.1145/3161184

\footnotetext{
Permission to make digital or hard copies of part or all of this work for personal or classroom use is granted without fee provided that copies are not made or distributed for profit or commercial advantage and that copies bear this notice and the full citation on the first page. Copyrights for third-party components of this work must be honored. Abstracting with credit is permitted. To copy otherwise, or republish, to post on servers or to redistribute to lists, requires prior specific permission and/or a fee. Request permissions from Permissions@acm.org.

Copyright ACM 2017 2474-9567/2017/12-ART127 \$15.00

https://doi.org/10.1145/3161184
} 


\section{INTRODUCTION}

Working dogs enhance and protect many human lives worldwide. The definition of assistance dogs includes guide dogs for people who are blind, hearing dogs for people who are deaf, and service dogs for people with physical disabilities [23]. Dogs are also trained for a variety of other working roles, such as medical alert, psychiatric service, and PTSD intervention. For example, medical assistance dogs are trained to alert at the onset of seizures, hypoglycemia, and even an autistic episode. As working members of society, dogs have also been shown to improve social integration and self-perception of those with disabilities, while additionally providing social and emotional support for their owners [14,22].

There are upwards of 20,000 assistance dogs currently actively working in the U.S. [26]. Depending on the type of assistance dog training required, preparing a dog for the rigor of his or her working role can take up to two years [24]. Considerable resources are invested in assistance dogs-in-training; it can cost up to $\$ 50,000$ to raise and train a dog, and many of the canine candidates are released before graduation, with estimates of up to a $60 \%$ failure rate. Identifying which dogs are likely to succeed and which are likely to fail as early as possible could save millions of dollars in time and resources.

A major source of failure is an unsuitable temperament; a working dog must be reliable, calm, safe, and under control at all times in public. U.S. ADA laws provide public access and protection for assistance dog teams [25], so it is critical that assistance dogs have an appropriate temperament as well as correct training. If we can assess and quantify salient aspects of a dog's temperament, we may be able to predict success as an assistance dog. Berns et al. [2] have been highly successful in predicting whether a dog in training will graduate by employing fMRI scans of awake dogs. This approach requires training the dogs to lie still in the magnet. To quantify canine interactions and activity, researchers have also investigated wearable sensors for activity monitoring [20]. However, this approach categorizes actions in terms of gross movement, such as "sitting" or "running." While this information is useful, being able to quantify finer levels of activity, such as how hard a dog is biting a toy and the frequency with which they bite when playing with a toy, could provide clues to a dog's temperament. Currently, there are no methods for quantifying those types of interactions. Our goal for this study was to develop sensors, in the form of instrumented (smart) dog toys, to investigate whether we can use natural canine object-play interactions to quantify temperament, and to predict outcomes of puppies in service dog training programs.

We were interested in exploring two main topics: (1) the design of sensor-enabled smart toys; and (2) the development of statistical models employing the smart toy data to predict the suitability of candidate CCI assistance dogs. We investigated two toy designs based on commonly available dog toys, a fetch toy (a ball) and a tug toy, developing and refining a classifier to analyze the sensor data and make predictions.

\section{BACKGROUND AND RELATED WORK}

\subsection{Sample Fabrication}

Current methods for determining aspects of temperament consist of a battery of behavioral tests that are performed by professional trainers, usually when the dogs are young puppies. Even though many organizations that breed their own dogs select for calm and even temperaments, there are no guarantees that the puppy will successfully be placed as an assistance dog. Early training, from both the puppy's mother and his human puppy-raiser, are shown to make an impact [5].

2.1.1 Behavior Testing. Canine behavior is a combination of temperament, a dog's innate drive and the patterns of behavior that exhibit themselves from infancy, and a dog's experience shaped by his environment, socialization, and training. Existing tools for assessing a dog's behavior, such as the Volhard Puppy Assessment Test (PAT) and the Canine Behavioral Assessment and Research Questionnaire (C-BARQ), allow us to determine various aspects of the canine temperament, such as excitability, aggressiveness, or sensitivity to different stimuli $[10,21]$. These tools, however, take time, skill, and resources to conduct, which are not 
always feasible for service dog programs. Furthermore, while behavioral tests can be indicative of suitability [6], the repeatability, reliability, and subjectivity of these studies has always been in question $[8,9,15,16]$.

2.1.2 Object-Play Behavior and Temperament. How dogs play reflects general attributes of their temperament and their relationship with their owner [19]. For example, solitary object play, such as when a dog plays with a toy by himself without a human, has been related to predatory behavior [3]. Additionally, studies show that social play involving objects held by a person are more interesting to a dog than the same object held by a mechanical device [18]. This difference is not surprising because play is often used as a reward in training, as play with familiar humans is a powerful reinforcer for dogs [1,7].

Observing object-play behaviors can provide insights into a dog's temperament. A dog's response to novel objects, such as a willingness to investigate, vocalization, or avoidance can illustrate the dog's confidence level and how he might react in an emergency. Call response times and the duration of a dog's interest in an object can provide information on a dog's cognitive abilities. Similar to human babies, cognitive capabilities such as understanding object permanence develops in dogs with age [4].

\subsection{Previous Work}

In the Facilitating Interactions for Dogs with Occupations (FIDO) project, we developed sensors that assistance dogs can activate to communicate with their human partners [11,12]. For example, a hearing service dog can activate a sensor on his service dog vest to tell his handler that the sound he just heard was a tornado siren, rather than the doorbell, allowing his human partner to make better choices about how to respond.

In the FIDO study, we created nine different sensor designs based on the natural abilities of dogs: biting, holding, tugging, and nose touching. One of the most interesting results of the FIDO project was our observation that the data from dog-activated sensors showed patterns that informally correlated with the known temperament of the test subject dogs. The graphs below show activation data for an on-body FIDO bite sensor from three different dogs. All three dogs were trained as service dogs, and one is currently an active service dog. The bite activations, cued by a verbal command from the handler, appear as spikes on the graph, and activations that cross the red threshold line activated the sensor (causing a feedback tone to the dog).

In the first graph (Fig. 1), the bites are very regular and consistent. This dog has an excellent temperament for service dog work. He is easily trained and has a very good work ethic; he always performs what is asked of him to the best of his ability, with minimal reward. His bites are consistent across the entire 140 second (s) timeframe. In the second graph (Fig. 2), we see that the bites are very timid and sporadic across a 300 second period. Although this dog was also trained for service dog work for over two years, his temperament was too "soft," and he was eventually released from the service dog program (after considerable investment in his training). In the third graph (Fig. 3), we see a multitude of bites within 100 seconds, with some extra (spontaneously offered) activations. This dog is an active medical alert service dog, but is very high-drive and is placed with an able-bodied person who is a professional dog trainer. He is a very successful service dog because of his drive and trainability, but he needs firm direction to stay on track.

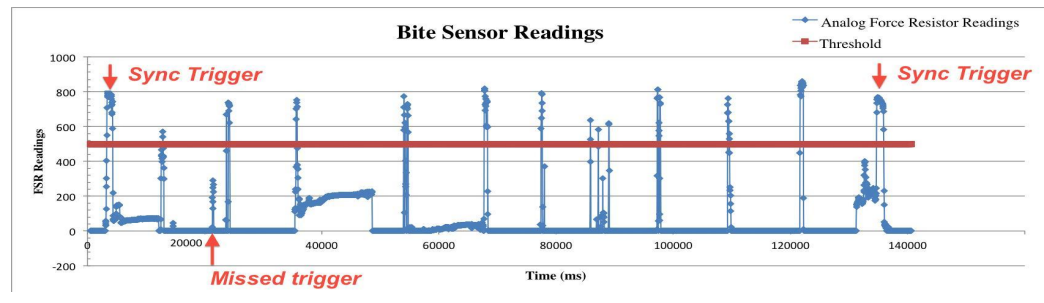

Fig. 1. Bite sensor graph for medium-drive dog. 


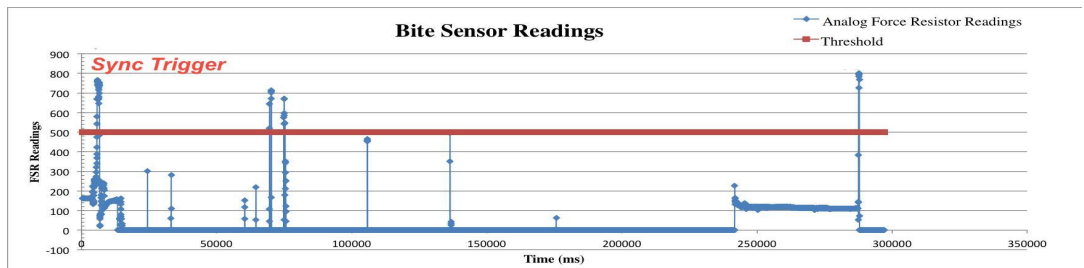

Fig. 2. Bite sensor graph for low-drive dog.

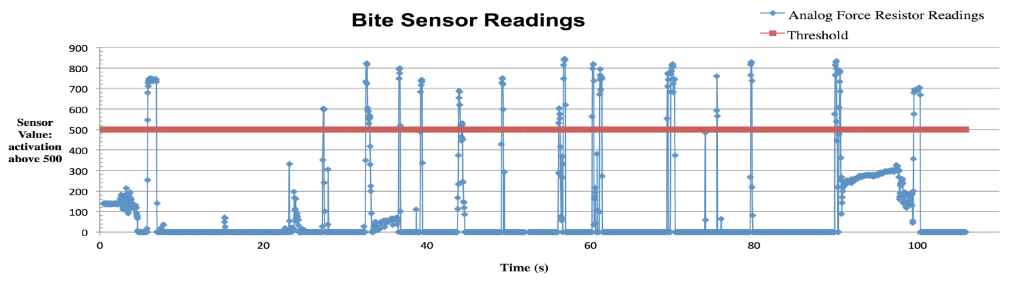

Fig. 3. Bite sensor graph for high-drive dog.

The observation that sensor interaction data may correlate with a dog's temperament was the inspiration for our main research question: to what extent can interaction with instrumented objects, such as our Smart Toys, predict aspects of temperament, and ultimately the success, of an assistance dog in training? In evolving our FIDO sensors for interaction, the ones that involved biting and tugging showed patterns that seemed to more clearly reflect the dogs' temperaments than the ones that involved proximity and touch. In addition, the retriever in our sensor study interacted correctly more often with the bite and tug sensors than with other sensors, seeming to better understand what to do with the ball- and tube-shaped devices, which makes intuitive sense given the breed. Thus, given our service dog population for this study would involve retrievers, we decided to focus on instrumenting bite and tug toys.

\section{INSTRUMENTED TOY DESIGN}

Our goal was to create devices that do not require training. Fetch and tug toys are two of the most common objects used in play between humans and dogs [19]. Consequently, we designed new sensors in the form of common toys with which many dogs naturally engage. We built a self-contained, ball-shaped sensor approximately the size of a tennis ball consisting of food-safe silicone. We also created a tug sensor, which is a bungee, rope-like design with a silicone rubber grip for the dog to bite down and tug. We designed both sensors so that they could be used with or without a human so we could test for changes in interaction with the instrumented toys when humans were not directly involved in the play. Prior research indicates that companion dogs prefer play activities and interactions that involve humans over asocial interactions [17]. Therefore, we wanted to be able to test between whether the toys with human interaction versus the toys alone could tell us anything about a dog's eventual success.

\subsection{Sensing Considerations}

The first part of designing and testing our instrumented sensors was to consider what sort of interactions and activities we wanted to measure. We considered a variety of choices, but we chose fetch interactions and tugstyle interactions, both of which are behaviors seen in dogs from when they are small puppies through adulthood. We believed that because these are such ubiquitous and natural dog behaviors, these sensors could be used across age groups, though the interactions for disparate ages might be different. Additionally, before conducting the full study at CCI's training center, we conducted an initial test to ensure that our sensors 1)

Proceedings of the ACM on Interactive, Mobile, Wearable and Ubiquitous Technologies, Vol. 1, No. 4, Article 127. Publication date: December 2017. 
collected the data we wanted, 2) were durable enough to last through the experiments, and 3) allowed us to accurately measure the interactions.

After testing our sensors extensively in our own lab, we conducted several test sessions through three informal "beta test" studies at Comprehensive Pet Therapy, a local pet training facility. Beta testing allowed us to ruggedize the sensors and fully vet our experimental protocol to prepare for our first round of testing at CCI. Because none of the dogs in this cohort were assistance dogs in training (although there were several released CCI dogs), building a predictive classifier would not be meaningful, so we did not perform the full data analysis with this initial cohort. However, this initial experimentation allowed us to better understand how dogs would interact with our sensors, ensure we could collect the data we needed for a full study, and find and mitigate flaws in our initial sensor designs. We were also able to experiment with our protocol to ensure that our method was sound and repeatable and to ensure that we did not experience data or video loss.

3.1.1 Design Tradeoffs. Designing the sensors involved balancing several considerations: the data we needed to collect, the size of the dogs we were testing, and the cost and technology involved. Based on our earlier research, we knew both force-sensitive resistors or pneumatic sensors could work well for measuring force $[11,12]$. We investigated methods of measuring bite force on both sensors, considering different methods of measuring the force applied to an object. We also wanted our sensors to be invariant to the direction of force applied, which meant that we would need several force sensitive resistors to measure bite depressions in several axes. The pneumatic bite sensor, relying on the air pressure within the sensor, could measure pressure along any bite axis or orientation as long as the sensor had a relatively soft shell. The shell design needed to be conducive to consistent replication for reliable data collection. We considered different methods of hollowing out tennis balls, or using bicycle tire tubing to make air-tight flexible sensors; however, the consistency was a challenge. Additionally, we wanted to ensure that the toys were safe for the dogs to bite and chew on, so we had to avoid anything that could potentially have toxic chemicals in it. We eventually settled on a food grade two-part silicone rubber (SHORE A37 hardness) that we could cast using 3D printed molds of the shapes we wanted. The pneumatic bite sensor, relying on the air pressure within the sensor, could measure pressure along any bite axis or orientation if the sensor had a relatively soft shell.

The next challenge was to embed the sensors into the toys. For the tug sensor, this task was not difficult because we could run a tube out of the bite sensor to a separate board. For the fetch sensor, we needed a toy that could be reliably and repeatedly "launched" for the dog to fetch. We settled on a ball sensor that could be rolled or tossed, allowing for flexibility in its use. However, for the ball sensor, the electronic components needed to be fully contained and very small so they would fit inside the ball. We considered a variety of approaches, investigating options for different forms of wireless charging, Bluetooth radios, and other techniques. Largely due to size constraints, we ultimately designed the ball sensor without any telemetry and instead recorded all the data to an SD card. The ball uses an IMU with a gyroscope, accelerometer, and magnetometer, as well as a separate barometer, to measure both pressure and movement changes of the ball. During beta testing, we found that during use the SD card could be unintentionally ejected causing a loss of data during a trial. We changed the placement of the SD card slot on the board so that when a SD card was plugged in, it did not overhang the outer edges of the board, which fixed the issue.

3.1.2 Goals for the Toys. The toys needed to be durable to last through many uses by at least several dogs without degradation. We also needed to be able to wash the toy between uses, cleaning any saliva or dirt that got on the toy during testing. The outer shell of the ball needed to protect the relatively simple electronics, which impacted how thick we had to make the exterior silicone walls of the sensor toy. Lastly, we needed to make sure we could get to the sensor to retrieve the data collected using the ball. Because we opted not to include wireless telemetry for size reasons, we designed the ball itself to be opened so we could access the internal SD card which recorded the data.

We designed the ball with a minimum set of specific behaviors we wanted to be able to measure, which impacted how we designed the electronics. First, we wanted to be able to measure the frequency and intensity of biting and chewing on the ball. Additionally, we wanted to measure other behaviors such as shakes and the duration of time the dog interacted with the fetch toy. Our main focus for the tug toy was to be able to Proceedings of the ACM on Interactive, Mobile, Wearable and Ubiquitous Technologies, Vol. 1, No. 4, Article 127. Publication date: 
measure tug force in addition to the features we were detecting for the ball. During testing, we adjusted the length of the tug toy to allow a wider bite area so that the dogs could get a more solid bite which seemed to impact whether the dogs would try to tug on the toy as well.

\subsection{Final Instrumented Toy Design}

Moving forward from our beta tests, we finalized a self-contained silicone ball, and a tug sensor, which is a silicone tube that can be tethered to an object in the environment or to a human.

3.2.1 Silicone Ball Sensor. The ball sensor is composed of an inner ball and an outer ball, both molded from silicone. As shown in Fig. 4, the inner ball (bottom) has an opening to accommodate inserting the electronics and has a locking mechanism to prevent the outer ball from rotating on the inner ball. The outer ball (top) has an opening to allow inserting the inner ball. The outer ball protects the electronics and provides an air space for the barometric pressure sensor to operate. When a dog bites the sensor, the air pressure inside the ball increases, and the electronics record the pressure on an SD card.

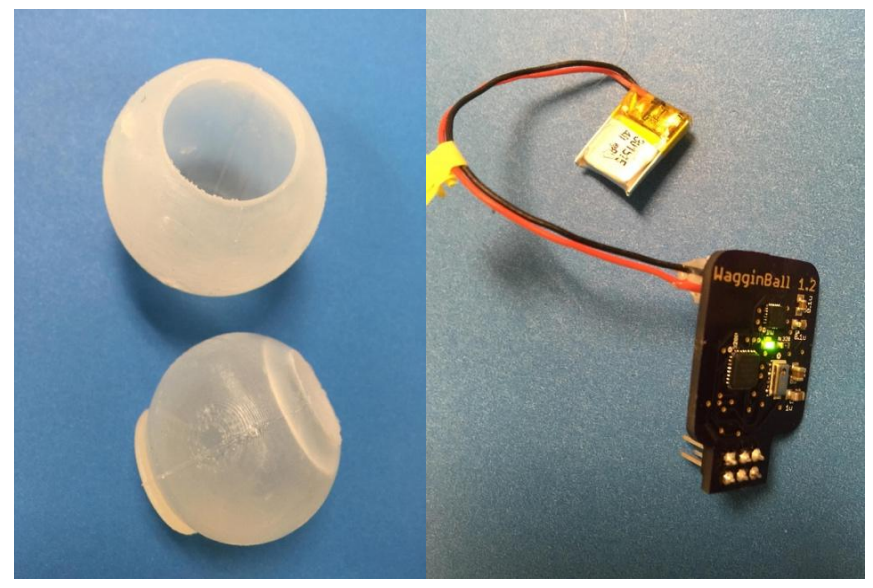

Fig. 4. Ball sensor. left: outer ball and inner ball; right: electronics and battery that are placed inside the inner ball.

The electronics consist of a custom board that we designed, which includes a barometer that can measure pressures ranging from 7.25 PSI - 16.68 PSI. From what we learned from testing with our local cohort of dogs, we revised and re-built the design for the ball sensor to be more robust and to better protect the electronics. We augmented the board to incorporate an accelerometer, gyro, and magnetometer in addition to the pneumatic sensor. The accelerometer allows us to capture some "kill behaviors" (shaking toy) as well as characterizing the intensity and duration of the dog's play behaviors. The gyro lets us measure movement "gestures," as well as detecting a rolling ball, and the magnetometer gives us the opportunity to perform a sync trigger with a small magnet useful for synchronizing our data collection with the video recording. Fig. 5 shows the electronics inside the inner ball being inserted into the outer ball, and then the assembled ball. Note that the translucence of the ball allows researchers to observe the green light inside, indicating that the board has power. We built two new boards for the ball sensors and six new silicone balls to prepare for our first test at CCI. 


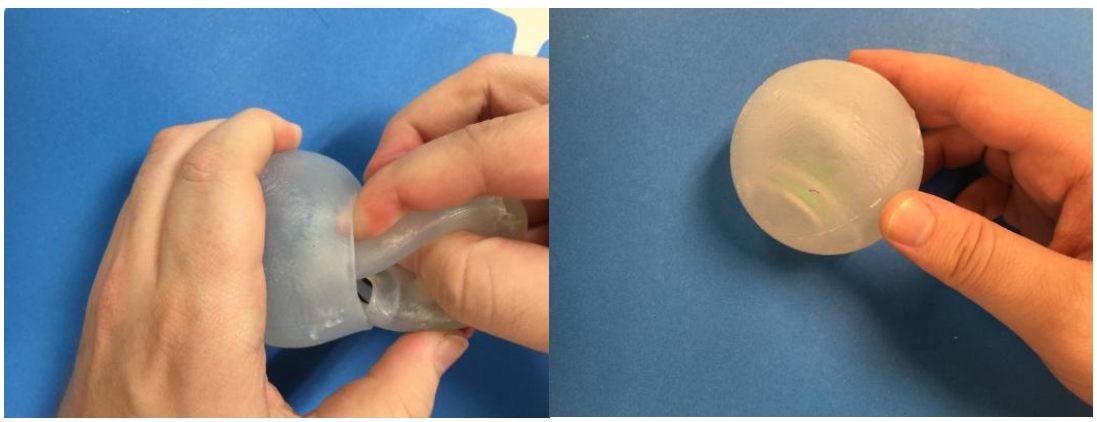

Fig. 5. Ball sensor. left: inserting the ball; right: assembled ball.

3.2.2 Silicone Tug Sensor. The second sensor we created is a tube shape attached at one end to an object in the environment or held by a human. This sensor also includes our custom board with a pneumatic sensor, which contains a barometer that can measure pressures ranging from 7.25 PSI - 16.68 PSI, as well as a stretchsensitive resistor, built into the handle, which measures the force of the dog tugging on it. The stretchsensitive resistor measures $1 \mathrm{k}$ Ohms-per-linear-inch of material, and the total resistance quadruples when stretched to twice the length. Fig. 6 shows the tug sensor and its handle. We also created simulated fur covers for the tug sensor, to test what materials dogs prefer to interact with.

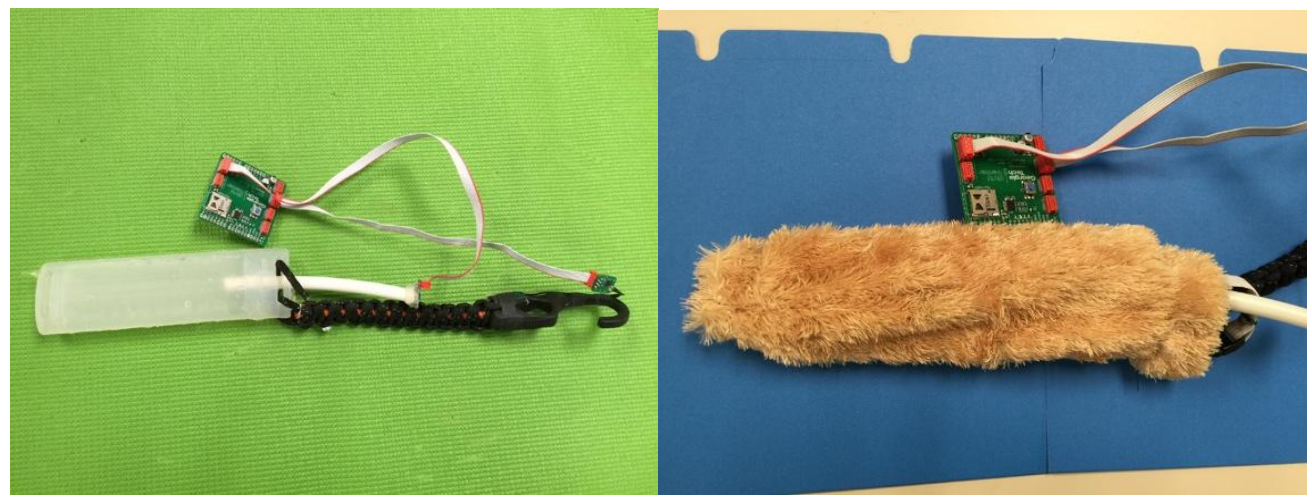

Fig. 6. Tug sensor: left: The silicone tube sensor, attached to its tug sensor handle, and the electronics (external); right: Tube sensor with simulated fur cover.

We created four new tube sensors for our study at CCI, and made fourteen covers for the sensors, in order have a new cover for each dog and remove scent bias, washing the covers between each data collection session.

\section{DATA COLLECTION AND EXPERIMENTAL SETUP}

\subsection{Participants}

We collected data from 40 dogs undergoing advanced training at the CCI facilities in Santa Rosa, CA during the same period as Berns et al. [2] (see Table 1). All dogs had basic obedience training and socialization. We tested 23 females and 17 male dogs between the ages of 17 and 21 months old. Of the 40 dogs, 1 was a Golden Retriever, 8 were Labradors, and 31 were Labrador-Golden Retriever crosses. These dogs were part of another 
study [2], which required the dogs to be trained enter an fMRI and be scanned. Thus, potential selection bias might have occurred due to the dog's ability to withstand loud noises of an fMRI and to be trained for this task.

Table 1. Demographics of Participants

\begin{tabular}{lcccc}
\hline \multicolumn{5}{c}{ Breed } \\
\hline Female & GLD & LAB & LGX & Sum \\
Male & 1 & 5 & 12 & 17 \\
Total & 1 & 3 & 19 & 23 \\
\hline
\end{tabular}

Of the 40 dogs, 10 were released due to behavioral reasons, which varied from excessive barking to fearfulness of riding an elevator. Dogs who successfully finished advanced training were placed in one of five categories. They could be placed as a skilled companion, a service dog, a hearing dog, a post-traumatic stress disorder (PTSD) dog, and a facility dog, each of which has varying levels of service dog skill involved. Table 2 shows the dog outcomes for the study.

A skilled companion typically has an even and calm temperament, is in great health, and has basic obedience training. They are placed with individuals who cannot manage a dog by themselves, such as children or non-independent adults with disabilities. A service dog assists with both physical tasks and provides emotional support for independent individuals with a disability. A hearing dog is trained to distinguish between different sounds for the hearing impaired. A PTSD dog is trained to help veterans who suffer from flashbacks or other PTSD-related conditions. A facility dog is trained to work with a professional therapist or teacher to help multiple individuals, such as at a school or therapy facility.

Table 2. Outcomes of the Working Dogs

\begin{tabular}{lc}
\hline Outcomes & $\#$ \\
\hline Service Dog & 17 \\
Skilled Companion Dog & 6 \\
Facility Dog & 4 \\
Hearing Dog & 1 \\
PTSD Dog & 2 \\
Behavioral Release & 10 \\
Total & 40 \\
\hline
\end{tabular}

\subsection{Description of the Data Collected}

4.2.1 Silicone Ball Sensor. The IC barometer collected data in kiloPascals and measured the changes and variations in internal pressure based on the ambient internal air pressure. The 9-axis IMU collected the changes in $\mathrm{X}, \mathrm{Y}$, and $\mathrm{Z}$ values of the gyroscope, accelerometer, and magnetometer.

4.2.2 Silicone Tug Sensor. Again, the IC barometer collected data in kiloPascals and measured the changes and variations in internal pressure based on the ambient internal air pressure. The stretch sensor detected the changes in resistance, measured in ohms, across a 3 inch stretch sensor. 


\subsection{Experiment Design}

We tested 40 dogs on each of the two sensors in a single session for each sensor.

4.3.1 Experimental Protocol: Part 1 - Single Trials. We traveled to Santa Rosa, CA four times, on May 8, 2015 (testing 14 dogs), July 17, 2015 (10 dogs), October 16, 2015 (12 dogs), and January 15, 2016 (12 dogs), testing a total of 48 dogs. Eight of these dogs were selected for other programs (breeding or medical alert) and were removed from our cohort, leaving us with $40 \mathrm{dogs}$. Some of the dogs were still in advanced training and some were already released (although we were blind to this information initially so as to not bias the experiment). We tested each dog with 10 trials of each of the four toy conditions in a randomized order, with at least 30 minutes of rest between:

1. Ball sensor, rolled by human (researcher)

2. Ball sensor, rolled down a ramp (machine)

3. Tug sensor, attached to researcher, no cover

4. Tug sensor, attached to researcher, faux fur cover

We performed 10 trials with each set of sensors for each dog, for a total of 40 trials per dog. Each run of 10 trials was video recorded, and after each run, both the device's battery was changed and the data and video were uploaded to determine if any loss occurred. If there was loss, we re-ran the missing trials. Each trial was video-taped from two perspectives. One camera was near the researcher, allowing us to review the trials from the perspective of the researcher, and another camera was focused on the researcher, to capture early interactions.

4.3.2 Silicone Ball Sensor Experiment. The silicone ball sensor experiment was conducted in a closed room, with the shades drawn, to prevent distraction from trainers and other dogs. Fig. 7 shows the experimental setup for the ball-human condition. Both conditions had a 15'x 4' wide section of the floor covered in $5 \mathrm{~mm}$ non-slip PVC mats so that, as the dogs ran after the ball, they wouldn't slide and hit the wall. Before and after each dog's session, we ran a magnet along the length of the ball so that we knew the exact start and finish times. After each session, the sensing board was removed from the silicone enclosure and the data was uploaded and removed.

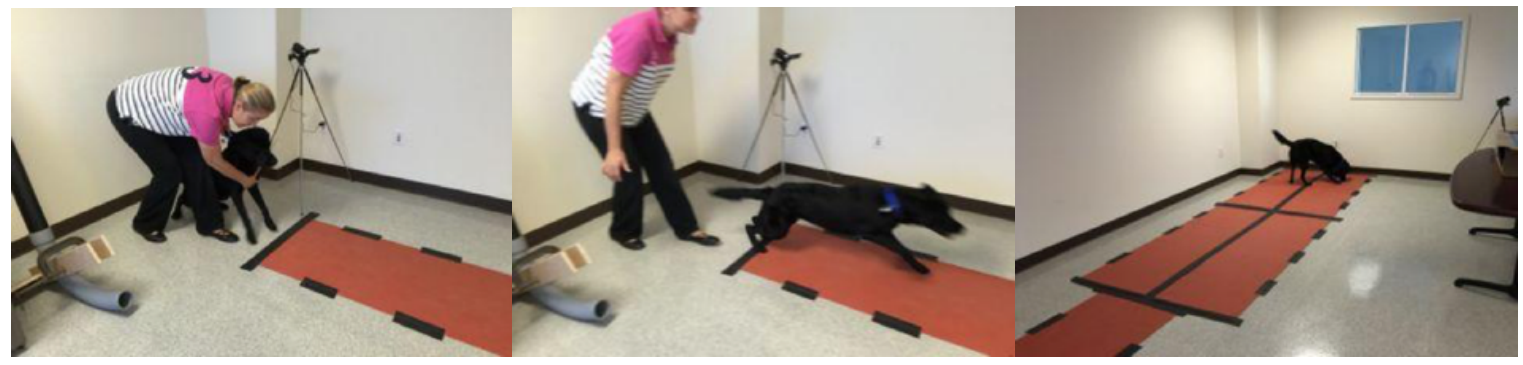

Fig. 7. Ball-Human Sensor experiment setup. The experimenter rolls the ball, and then (left) the dog is mildly restrained for one second, and (middle) released to pursue the ball. The dog then retrieves and interacts with the ball (right).

Ball rolled by stranger. For the first condition, a researcher unfamiliar to the dogs stood at the end of the "runway" and rolled the ball towards the other end, holding the dog back for one second.

Ball released by machine / ramp. For the second condition, the dog started each trial next to a ramp, which was a tube with a curved end constructed of PVC pipe (shown on the left of the leftmost photo in Fig. 7). Researchers put the ball into the ramp and released it, simultaneously releasing the dog. 
4.3.3 Silicone Tug Sensor Experiment. Again, the experiment was conducted in a closed room, with the shades drawn, to prevent distraction from trainers and other dogs. As shown in Fig. 8, the researcher presented the silicone tug sensor to the dog at hip height with the verbal cue "Get it." After any interaction with the sensor for more than 10 seconds occurred, the dog was rewarded with a "Good [boy/girl]" and a "release" command. Each dog was asked to interact with the toy 10 times. However, if the dog lost interest in the task completely (refused to "get it"), then the study was concluded early. After each dog's session, the data was removed from the SD card and stored appropriately.

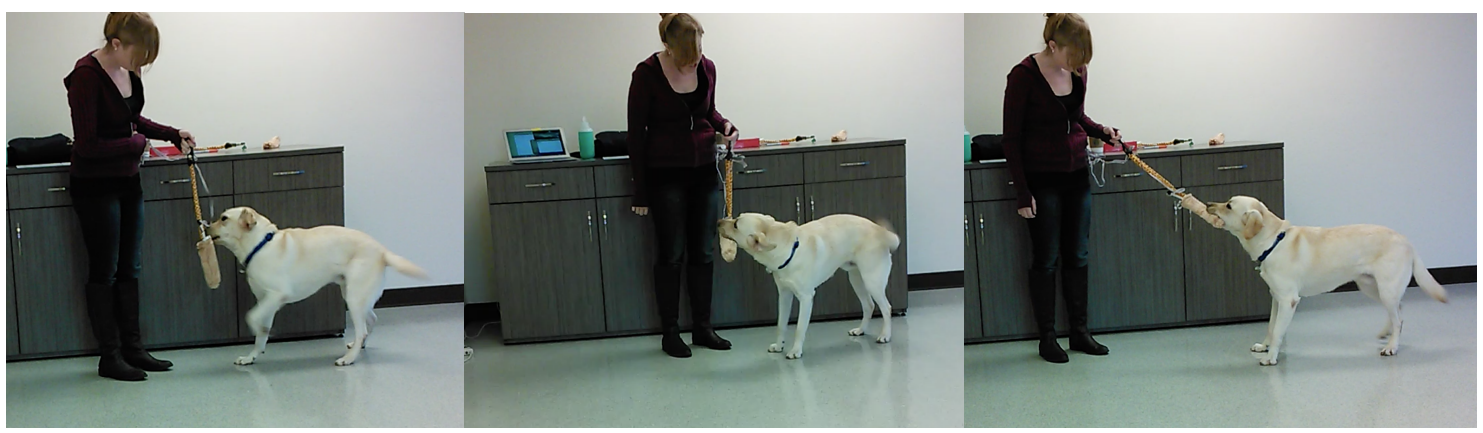

Fig. 8. Covered Tug Sensor experiment setup. The experimenter holds the tug sensor out for the dog (left), the dog bites down on the tug sensor (middle), and the dog tugs on the toy (right).

\section{PREDICTION FRAMEWORK}

\subsection{Feature Construction}

We constructed 22 features from the raw data of each of the ball conditions and 10 features from each of the silicone tug sensor conditions. We constructed these features from observations from previous generic canine interactions.

5.1.1 Silicone Ball Sensor. We calculate a core set of features and then determine the average, maximum, and total numbers during a trial. Using those numbers, we also calculate the trend of these numbers across a session, calculating the difference between the first and last trials in each session.

Our core features include:

1. Interaction Time: the amount of time required for a single trial, from the time the ball rolled out of the ramp or hand until the dog retrieved the ball to the handler

2. Number of Bites: the number of times the pressure crosses a threshold during each trial in a dog's session.

3. Bite Strength: the peak pressure throughout the duration of a bite

4. Bite Duration: the duration of a bite

5. Bite Frequency: number of bites in a trial, divided by the length in seconds of that trial

6. Bite RMS: the root mean square of all pressure samples throughout the duration of a bite

7. The Number of Active Trials: the number of trials within a session in which more than zero bites were recorded

8. The Number of Inactive Trials: the number of trials within a session in which zero bites were recorded

5.1.2 Silicone Tug Sensor. We calculate a core set of features and then determine the average and maximum of these features.

Proceedings of the ACM on Interactive, Mobile, Wearable and Ubiquitous Technologies, Vol. 1, No. 4, Article 127. Publication date: December 2017. 
Our core features are:

1. Covered/Uncovered: did the sensor have the cover on it

2. Number of Bites: the number of times the pressure crosses a threshold during each trial in a dog's session

3. Bite Strength: the peak pressure throughout the duration of a bite

4. Bite Duration: the duration of a bite

5. Number of Tugs: the number of times the resistance crosses a threshold during each trial in a dog's session

6. Tug Strength: the peak stretch resistance throughout the duration of a tug

7. Tug Duration: the duration of a tug

\subsection{Classification Model Goals}

Given the goal of optimizing overall costs of dog training by making a decision on which dogs continue to advanced training after puppy raising, we attempt to choose thresholds for our classifiers to maximize the value of the equation:

Value $=\mathrm{TP}^{*} \$ 19,000-\mathrm{FP} * \$ 17,000-\mathrm{FN} * \$ 2,000$,

where TP are true positives - dogs that the classifier correctly predicts will be successful in advanced training. These numbers are based on CCI's Annual Report [27] and do not include the personal costs to the volunteer puppy raisers. The estimated cost of the training of these dogs is $\$ 2,000$ through puppy raising and an additional \$17,000 through advanced training and placement. Thus, we place the value of a successful dog at $\$ 19,000$. FPs are false positives - dogs that the classifier predicted would be successful in advanced training that were not. Each false positive wastes $\$ 17,000$ (assuming that an early failure of a dog still wastes advanced training resources that could have been applied to a successful dog). FN are false negatives - dogs that the classifier predicted would fail advanced training even though they would have been successful. False negatives waste $\$ 2,000$ from puppy raising. True negatives are dogs which the classifier correctly predicts will not pass advanced training. As the classifier is applied before advanced training and not before puppy raising, there is no cost savings possible for true negatives. This analysis assumes that there is a constant supply of dogs coming through the puppy raiser process, that advanced training is a limited resource, and that the cost of testing and the cost of placing unsuccessful dogs in homes are minimal.

To give an example, this study follows 40 dogs. All were passed into advanced training, and 10 were released at various points during their advanced training (failed), while the remaining 30 were given a working dog role and placed with someone in need. This result is equivalent to a classifier where there were 30 true positives, 10 false positives, and zero false negatives. Thus, the return value of the current process (in this case, training all dogs that pass the inclusion criteria for the fMRI study) is $\$ 400,000$.

Value $_{\text {CCI_Current }}=30 * \$ 19,000-10 * \$ 17,000-0 * \$ 2,000=\$ 400,000$,

A perfect classifier would raise the return to $\$ 570,000$, saving $\$ 170,000$ in un-utilized advanced training. For optimal results, we seek a method of testing the dogs such that the maximum number of true positives are returned while simultaneously false positives are penalized at a factor of 8.5 to 1 over false negatives $(\mathrm{FP}=$ $\$ 17,000$ : $\mathrm{FN}=\$ 2,000$ ). For any method below to be an improvement over current practice for the cohort under study, it must have classified at least 23 of the 30 successful dogs correctly with no false positives $(23$ * $\$ 19,000$ - $0 * \$ 17,000-10 * \$ 2,000=\$ 417,000)$. Any increase in false positives would require more true positives to offset the increased deficit. Thus, we only need examine the true positive vs. false positive (ROC or receiver operating characteristic) curves for regions above $77 \%$ (23/30) true positives. 


\subsection{Classification Model}

We frame prediction as a binary classification problem, where we discriminate between passing and failing advanced training. For the purposes of classification, we represent each dog as a vector of features, where the vector consists of the dog's gender, 22 features from the ball-human trials, 22 features from the ball-ramp trials, 10 features from the covered tug trials, and 10 features from the uncovered tug trials.

We compared several different parametric and non-parametric classifiers to empirically determine the most suitable classification technique, including random forests, decision trees, Naïve Bayes, and Logistic Model Tree (LMT). We use independent training and test sets for 10 -fold cross-validation. Specifically, we randomly select four dogs for testing and train on the remaining 36. There is no testing on training during cross validation. We use six different performance metrics: accuracy, precision and recall, $\mathrm{F}_{1}$ score, Matthews correlation coefficient, and the area under the curve (AUC). While Random Forest appeared to perform just as well as LMT, it's error rate consisted predominantly of false positives, with 8 false positives and 1 false negative. As the goal of our classification model was to prevent false positives, we found that the best performing classifier was LMT. LMT is a classification model that combines logistic regression and decision tree learning. LMT uses a Classification and Regression Tree (CART) cost complexity pruning method. To reduce our feature set, we ran feature selection across an aggregated dataset to determine the most valuable features. Again, we leverage independent training and testing sets for each fold during cross-validation. From the 65 initial features, we selected the five most discriminative features: maximum number of bites in the ballhuman condition, the average and maximum number of bites in the ball-ramp condition, the average duration of bites in the ball-ramp condition, and the average tug duration for the uncovered tug toy condition.

Table 3. Performance Metrics of Attempted Classifiers in Predicting Working Dog Placement

\begin{tabular}{llccccc}
\hline & \% Accuracy & Precision & Recall & F & MCC & AUC \\
\hline LMT & $77.5 \%$ & 0.839 & 0.867 & 0.852 & 0.380 & 0.727 \\
\hline Random Forest & $77.5 \%$ & 0.784 & 0.967 & 0.866 & 0.274 & 0.807 \\
\hline Naïve Bayes & $75.0 \%$ & 0.500 & 0.700 & 0.583 & 0.424 & 0.815 \\
\hline Decision Tree & $72.5 \%$ & 0.462 & 0.600 & 0.522 & 0.339 & 0.667 \\
\hline
\end{tabular}

\subsection{Outcomes and Explanatory Variables}

Using feature selection, we found that the most contributory variables were the maximum number of bites in the ball-human condition, the average and maximum number of bites in the ball-ramp condition, the average duration of bites in the ball-ramp condition, and the average tug duration for the uncovered tug toy condition. We use these five features for prediction.

\section{RESULTS OF PREDICTION}

The goal of this particular model is to optimize for the value equation defined above. However, we are also concerned about efficiency of testing and whether our learned model will generalize well versus overfitting. Extracting the five most valuable features using feature selection is a first step to both of these goals. Less features mean less chance of overfitting and may show that some of our testing is superfluous. We average across 20 runs of randomized 10-fold cross-validation, leaving 4 dogs out, using LMT and achieve 87.5\% accuracy. More metrics averaged over the 20 runs are presented in Table 3. Additionally, since our goal is to optimize for the Value equation, we provide the confusion matrix for our classifier in Table 4.

Table 3. Mean Performance Metrics in Predicting Working Dog Placement 


\begin{tabular}{lccccc}
\hline \% Accuracy & Precision & Recall & F & MCC & AUC \\
\hline $87.5 \%$ & 0.872 & 0.875 & 0.873 & 0.657 & 0.880 \\
\hline
\end{tabular}

Further evidence of good performance of this classifier can be found by looking at the ROC curve, achieving an AUC score of .88, as shown in purple in Fig.9. These curves plot the relationship of the true positive rate (dogs who we predict will be placed who actually get placed) against the false positive rate (dogs we predict will get placed who actually do not get placed) for different thresholds.

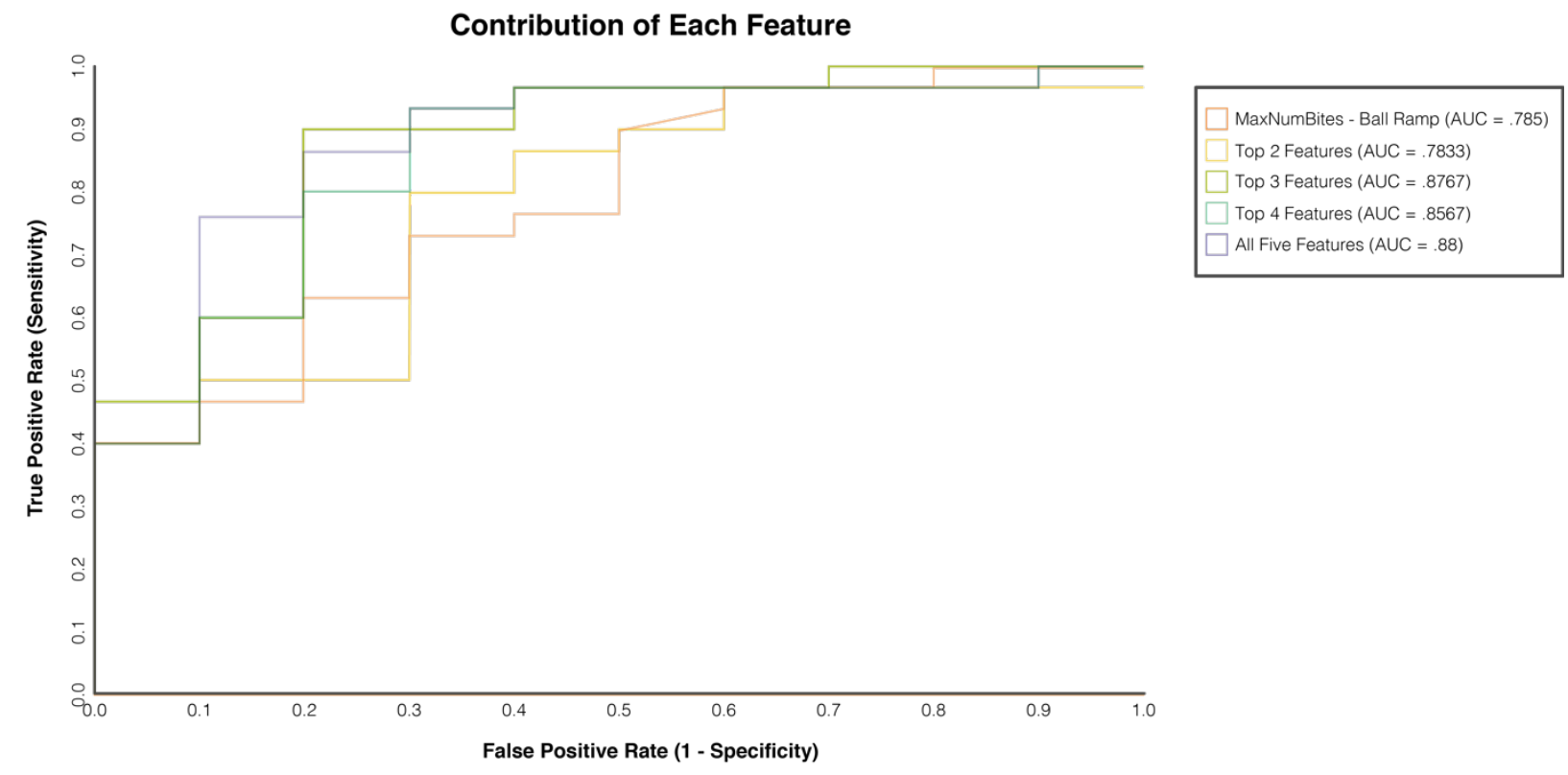

Fig. 9. Individual Feature Performance Metrics in Predicting Working Dog Placement (ROC Curves)

Table 4. Confusion Matrix for Predicting Working Dog Placement (rounded to the nearest whole number)

\begin{tabular}{|c|c|c|c|}
\hline & Predicted Pass & Predicted Fail & \\
\hline Actual Pass & 28 & 2 & $30(93.3 \%)$ \\
\hline Actual Fail & 3 & 7 & $10(70.0 \%)$ \\
\hline & $31(90.3 \%)$ & $9(77.8 \%)$ & \\
\hline
\end{tabular}

\subsection{Individual Feature Performance}

Above we use five features derived from the four different ball or tug sensor tests to achieve the best accuracy. Might we achieve similar results with less features and be able to avoid running all four types of tests? Using the individual features, we average across 20 runs of randomized 10-fold cross-validation using LMT and show their individual performance. Again, we use six different performance metrics: accuracy, precision and recall, $F_{1}$ score, Matthews correlation coefficient, and the area under the curve (AUC). These results are presented in Table 5. 
Table 5. Individual Feature Performance Metrics in Predicting Working Dog Placement

\begin{tabular}{llccccc}
\hline & \% Accuracy & Precision & Recall & F & MCC & AUC \\
\hline $\begin{array}{l}\text { Max \# Bites - Ball } \\
\text { Ramp }\end{array}$ & $82.5 \%$ & 0.821 & 0.825 & 0.803 & 0.480 & 0.798 \\
\hline $\begin{array}{l}\text { Avg \# Bites - Ball } \\
\text { Ramp }\end{array}$ & $80 \%$ & 0.792 & 0.800 & 0.766 & 0.385 & 0.780 \\
\hline $\begin{array}{l}\text { Max \# Bites - Ball } \\
\text { Human }\end{array}$ & $75 \%$ & 0.563 & 0.75 & 0.643 & 0.000 & 0.500 \\
\hline $\begin{array}{l}\text { Avg Bite Duration - } \\
\text { Ball Ramp }\end{array}$ & $72.5 \%$ & 0.679 & 0.725 & 0.690 & 0.131 & 0.510 \\
\hline $\begin{array}{l}\text { Avg Tug Duration - } \\
\text { Uncovered Tug }\end{array}$ & $72.5 \%$ & 0.558 & 0.725 & 0.630 & -0.092 & 0.467 \\
\hline
\end{tabular}

The maximum number of bites in the ball ramp condition achieves $82.5 \%$ accuracy, while the average performance of the other features individually provide less accuracy. Figure 9 shows the false positive versus true positive rates when the top $\mathrm{N}$ features are used for creating the classifier.

\subsection{Model Reliability Pilot Study}

To demonstrate the potential reliability and stability of our model, we collected 10 separate sets of data over time for each of the four toy conditions using a single dog known to be suitable as a service dog. The data was collected over a one-and-a-half-year period (see Table 6). While we do not use the Covered Tug data in our analysis, since it is not used in any of our five features, we still collected the data. All 40 dogs' data was used in the creation of the classifier for this test. However, this dog's data was not used at any point in the experimental training or tuning of the LMT classifier described above. The model predicts the dog would pass $100 \%$ of the time. We provide the model's confidence level in its prediction as well for each of the 10 sets of data. On average, the algorithm is over $80 \%$ confident in its prediction. 
Table 6: Dates on which we collected data from the dog used in our reliability pilot study. In 10 separate tests, the classifier predicts the success of a hitherto unseen service dog correctly.

\begin{tabular}{|l|l|l|l|l|l|l|}
\hline Session & Ball-Human & Ball-Ramp & Covered Tug & Uncovered Tug & Prediction & Confidence \\
\hline 1 & $09 / 30 / 2015$ & $09 / 30 / 2015$ & $10 / 07 / 2015$ & $10 / 07 / 2015$ & Passed & 0.844 \\
\hline 2 & $09 / 30 / 2015$ & $10 / 07 / 2015$ & $10 / 07 / 2015$ & $10 / 07 / 2015$ & Passed & 0.85 \\
\hline 3 & $09 / 30 / 2015$ & $10 / 14 / 2015$ & $10 / 14 / 2015$ & $10 / 14 / 2015$ & Passed & 0.891 \\
\hline 4 & $10 / 07 / 2015$ & $10 / 14 / 2015$ & $02 / 17 / 2016$ & $02 / 17 / 2016$ & Passed & 0.584 \\
\hline 5 & $10 / 14 / 2015$ & $10 / 14 / 2015$ & $02 / 17 / 2016$ & $02 / 17 / 2016$ & Passed & 0.874 \\
\hline 6 & $4 / 12 / 2017$ & $10 / 14 / 2015$ & $07 / 21 / 2016$ & $07 / 21 / 2016$ & Passed & 0.876 \\
\hline 7 & $4 / 12 / 2017$ & $06 / 22 / 2016$ & $07 / 21 / 2016$ & $07 / 21 / 2016$ & Passed & 0922 \\
\hline 8 & $4 / 12 / 2017$ & $06 / 22 / 2016$ & $07 / 29 / 2016$ & $07 / 29 / 2016$ & Passed & 0.791 \\
\hline 9 & $4 / 12 / 2017$ & $06 / 22 / 2016$ & $07 / 29 / 2016$ & $07 / 29 / 2016$ & Passed & 0.803 \\
\hline 10 & $4 / 12 / 2017$ & $06 / 22 / 2016$ & $07 / 29 / 2016$ & $07 / 29 / 2016$ & Passed & 0.864 \\
\hline
\end{tabular}

\section{DISCUSSION}

\subsection{Analysis}

Each of the 40 dogs performed the tests on all four toy conditions in a single day. While we randomized the order in which the dogs underwent each toy condition, it is possible that fatigue could have affected the dogs' stamina throughout the day. Looking at the five most valuable features that were derived from the feature selection analysis and the fact that they pull features from three out of the four experimental conditions imply consistency. Perhaps there is a relationship between a dog's stamina and their success as a working dog.

Furthermore, looking at the results in Table 5, we see that discriminating based on the maximum number of bites feature when we remove the handler from the equation (ball-ramp as opposed to ball-human) achieves $82.5 \%$ accuracy, while the other features appear to boost our accuracy and precision. If there is a relationship between a dog's stamina and their success, is it possible to simplify testing by using only multiple trials of the ball-ramp experiment and achieve the same or better accuracy? Further research will answer these questions.

A classifier that simply predicted that all dogs would be placed would result in $75 \%$ accuracy (misclassifying the 10 of 40 dogs that were not placed). Thus, an $87.5 \%$ accuracy would not seem to be too impressive. However, the costs of a false positive (classifying that a dog will succeed when it does not) far outweighs the costs of a false negative. The value in our classifier lies in its ability to predict which dogs will not be placed, as shown below. Using the equation in Section 5.3 discussing the optimization of our classifier, we can determine the cost benefits of using instrumented dog toys for predicting the suitability of CCI working dogs. Given that we correctly predict 28 out of the 30 dogs who were placed, have 3 false positives, and have 2 false negatives, the ending value of our 40-dog cohort would have been:

Value $_{\text {Toys }}=0.933 * 30 * \$ 19,000-0.300 * 10 * \$ 17,000-0.067 * 30 * \$ 2,000=\$ 476,790$, which provides significant utility over current practice $(\$ 476,790-\$ 400,000=\$ 76,790)$. 
In 2015, CCI placed 309 service dogs. At a $40 \%$ pass rate, that means that approximately 463 dogs went through portions of advanced training and were not placed, with 772 total dogs undergoing advanced training. At that current rate, it means that CCI is spending up to $\$ 7,871,000$ (463 dogs $\times$ $\$ 17,000$ ) a year on dogs that will not be placed. If the classifier described above was used on CCI's 2015 cohort to predict which dogs should not continue to advanced training, how much money would CCI have saved?

Calculating the value of the CCI 2015 cohort assuming all 772 dogs were given full advanced training and only 309 dogs were successfully placed yields.

Value $_{\text {CCI_CURRENT }}=309 * \$ 19,000-463 * \$ 17,000-0 * \$ 2,000=-\$ 2,000,000$,

which reflects the significant investment $\mathrm{CCI}$ makes with each cohort. If CCI were a for-profit company, the proposed value of each successful dog would have to be padded by almost $\$ 6500(\$ 2,000,000$ loss split over 309 successful dogs) to absorb the loss. However, the classifier, if it generalizes, might correctly predict a large majority of the dogs that will not succeed in being placed while suggesting that most of the dogs that will be placed successfully continue in training. Identifying these unsuccessful dogs early would have significantly changed the value of the cohort:

$$
\text { Value }_{\text {Toys }}=0.933 * 309 * \$ 19,000-0.300 * 463 * \$ 17,000-0.067 * 309 * \$ 2,000=\$ 3,074,937 \text {, }
$$

Thus, the classifier may have had to potential to increase the value of the 2015 cohort by $\$ 5,074,937$, mainly by reducing unnecessary advanced training costs! While it is unlikely the classifier will successfully identify $77.8 \%$ of the dogs that will not be placed (as suggested by the equation above), even identifying a small percentage of dogs unlikely to be successful would significantly affect the current economics of training service dogs (as long as the classifier does not improperly label too many dogs as failures that would be successful with advanced training). Clearly, this line of research is promising!

7.1.1 Performance of Maximum Number of Bites in the Ball-Ramp Condition. When selecting features, overfitting is always a risk. However, in the details above, one feature (maximum number of bites in the ballramp condition) provided most of the discrimination power between the classes. We provide the ROC curve and the confusion matrix using just this single feature in orange on Fig. 9 and Table 8. Even with a one feature classifier, the system would increase the value of the tested CCI cohort $\$ 47,000$ per year.

Value $_{\text {MaxNumBites_BallRamp }}=(29 * \$ 19,000)-(6 * \$ 17,000)-(1 * \$ 2000)=\$ 447,000$,

$\$ 447,000$ with classifier $-\$ 400,000$ current practice $=\$ 47,000$ in savings

The five features selected in the full classifier are surprisingly similar. Perhaps, as stated above, the addition discrimination power comes from repeated testing of a dog throughout the day. If this situation is true, testing could be performed with the ball-ramp condition alone, reducing the cost and complexity and improving the consistency of testing.

Table 8. Confusion Matrix for the Max \# Bites - Ball Ramp Feature for Predicting Working Dog Placement

\begin{tabular}{|c|c|c|c|}
\hline & Predicted Pass & Predicted Fail & \\
\hline Actual Pass & 29 & 1 & $30(96.7 \%)$ \\
\hline Actual Fail & 6 & 4 & $10(40.0 \%)$ \\
\hline & $35(82.9 \%)$ & $5(80.0 \%)$ & \\
\hline
\end{tabular}

7.1.2 Model Reliability. As stated earlier, on average, the algorithm is over $80 \%$ confident in its prediction of passing when tested on an unseen dog who is already known to be suitable as a service dog. However, trial 4's confidence level is 0.584 , the lowest recorded. During the time of trials 4 and 5 , the participant had an unnoticed (and untreated) Staph infection. Perhaps the score returned by the classifier might be used to 
determine the readiness of dogs on a particular day to do their work or as a measure of a dog's health over time?

7.1.3 Correlations between Breed, Dog Outcomes, and the Classifier. We also looked to see if there were any meaningful correlations between a dog's breed, their outcome, and our model's performance. As expected, the model fails on similar sets of dogs each time we randomized it. On average, out of the 5 dogs that the model misclassifies, all are lab-golden mixes (LGX), three were released, one was a skilled companion, and one was a service dog. Since 32 dogs are lab-golden mixes, 17 dogs become service dogs, and 6 of the dogs become skilled companions, misclassifications are more likely among these groups. Without a larger sample size, no real conclusions to breed-specific or outcome-specific classification correlations can be drawn.

\subsection{Instrument Dog Toy Limitations}

Our initial experiments at CPT highlighted situations in which the sensors could fail and allowed us to make them more robust. Initial problems included:

7.2.1 Silicone Failure. Initially, dogs could bite hard enough to puncture the silicone and consequently damage the electronics inside. We experimented with different densities and thicknesses of silicone and discovered that a harder silicone prevented the ball from being punctured. We used the same silicone density and hardness for all of the testing in the CCI study. Further investigation would be warranted if testing higher-drive, more powerful breeds such as Malinois or German Shepherd dogs used for police work.

7.2.2 Hardware Failure. In general, the hardware did not fail unless the ball was punctured. However, we did have a few instances of the battery being unplugged, or the SD card being ejected by bites that perfectly aligned with those junctures. We solved this by wrapping the inner electronics in soft fabric to cushion them and to keep them from moving around inside the inner ball

7.2.3 Inner Ball Rotation. Some of the harder-biting dogs were able to compress the outer ball enough that the inner ball could rotate inside of it, allowing the opening to be exposed. This problem was exacerbated by the fact that the dog's saliva could lubricate the two pieces of the ball to allow them to slip more easily, so dogs that interacted longer were more likely to rotate the inner ball. This problem could be solved by a fullycontained ball, described in future work below.

\subsection{Prediction Framework Limitations}

Reflecting on our process, there are many possibilities for improvement:

7.3.1 Simple Thresholding Techniques Versus Whole Activity Events. During beta testing, an initial analysis was conducted to determine the threshold for a "bite" and a "tug," where we attempted to choose an appropriate threshold that captured the majority of the bites. One could imagine using our current database to train a template matcher using dynamic time warping and k-nearest neighbors to better identify bites of different strengths. Going further, we could identify particular patterns of ball interaction, such as double bites, holds, shakes, or times that a dog attempted a bite and missed. We could also look for changes in bite strength, number of bites, or speed of acquiring the ball that might indicate fatigue or waning interest. Adding these features might prove fruitful in improving the accuracy of the system or in identifying differences across breed.

7.3.2 Inherent Bias of Feature Construction. Our analysis only included the constructed features that we knew we could measure and therefore have an inherent bias due our perceived notion of the relationship between the Smart Toys and our knowledge of the type of training the dogs would undergo. This approach proved to be successful, however, we acknowledge that other features may exist that could improve the accuracy of predicting a working dog's suitability. When investigating the use of Smart Toys for alternative 
types of working dogs (for example, allergen-detection or a police dog), the specific features we constructed may not be as successful.

\subsection{Future Work}

7.4.1 Fully Enclosed Silicone Ball Sensor. We completed all testing with the original nested-ball sensor design for continuity. However, we have been designing and experimenting with a fully-enclosed silicone ball that would be superior for "real world" use. This improvement would minimize damage to the internal components and speed testing, because the ball would not need to be disassembled to upload the data and change the battery.

7.4.2 Wireless Data Transmission. A fully enclosed ball requires wireless data transmission, although it does not need to be real-time as the dog interacts with the sensor. However, it needs to be fast enough to upload the data from the ball quickly after the session is completed, and needs to transmit all of the data reliably. We performed a survey of currently available wireless data transmission protocols and hardware currently available commercially. IRDA (infra-red) was a prime candidate but availability of parts is limited. After analyzing our speed and integrity requirements, we determined that Bluetooth is the best choice. It is ubiquitous, relatively low cost, and meets the speed and reliability requirements. We are currently augmenting our sensor board (with accelerometer, gyro, magnetometer, and barometer) with Bluetooth data transmission.

7.4.3 Inductive Charging. The other major challenge of a fully enclosed sensor is wireless charging of the battery. We performed further testing on the inductive charging circuit that we designed (charging the battery through silicone), and we are pleased with its capabilities. Our circuit design will eventually allow the ball to be wirelessly charged using a standard off-the-shelf Qi wireless charger. We have been designing ways of minimizing the footprint of the circuit design and incorporating it into the existing circuit board for the ball. The main issue currently is identifying rechargeable batteries that can withstand the temperatures needed for curing silicone (100 degrees Centigrade). Another approach is to create regions of conductive silicone so that the power receiving antenna is part of the ball shell itself or, alternately, the battery can be charged simply by attaching a charger to the ball's surface. We molded a silicone ball impregnated with graphite to make it conductive, and discovered that the silicone would not cure. Experiments with different kinds of silicone, and conductive materials, will be required to solve this issue. A final alternative is to create an inexpensive, disposable system that requires such low power that it can be used for months before being discarded. Initial analysis shows this approach is viable.

7.4.4 Additional Testing to Boost Accuracy and Increase Generalizability. To further refine and verify Smart Toys, we intend to continue testing what we have discovered on new cohorts of dogs. As mentioned earlier, the cohort we tested required training to enter an fMRI and to participate in being scanned. Given that the normal graduation rate is $40 \%$ and that the graduation rate of this set was $75 \%$, we wonder if the ball could help reject dogs in the average cohort of CCI candidates even more accurately than suggested in the testing above. Investigating dogs in other service programs would allow us to extend the generalizability of this framework to a wider class of working dogs.

7.4.5 Leveraging Activity Recognition to Understand the Quality of each Interaction and How it Changes over Time. Our goal for this study was to initially examine what it would take to predict the success and failure of service dogs. Opportunities exist to dive deeper and investigate how a canine's interactions vary across different temperaments, For example, do higher drive dogs have different bite patterns than dogs with a lower drive? Prompted by the results of the longitudinal study where our known suitable-for-service dog performed significantly differently on the test the day he was sick, we are also intrigued by possibility of using changes in our features to determine the ongoing health of an individual dog or their ability to perform their duty on a given day. 


\section{CONCLUSION}

We have found that dog toys instrumented with sensors to quantify a canine's object-play behavior can be used to make predictions about the dog's suitability to be an assistance dog. In the course of this work, we created new tools and evaluation techniques for detecting specific canine behaviors. We also constructed a novel methodology for building and evaluating predictive models that forecast the suitability of puppies successfully completing advanced training. Exploring outputs from the sensors allowed us to identify various features that are more valuable for the prediction of a service dog and we used these features to construct a predictive model with $87.5 \%$ accuracy. The potential for instrumented dog toys and our predictive tools could be significant for helping service dog organizations reduce the cost of training puppies, increase the efficiency of their programs, and enabling trainers to spend more time developing puppies with temperaments more suitable for working dog careers.

\section{ACKNOWLEDGMENTS}

This material is based upon work supported by the Army Contracting Command and DARPA under Contract No. W911NF-14-C-0094 to Dog Star Technologies, LLC. Any opinions, findings and conclusions or recommendations expressed in this material are those of the authors and do not necessarily reflect the views of the Army Contracting Command and DARPA.

We would like to express our sincere appreciation to our sponsor, Dog Star Technologies; we thank Greg Berns, Mark Spivak, Andrew Brooks, and Marian Scopa for their support during this research.

\section{REFERENCES}

[1] Gregory S. Berns, Andrew M. Brooks, and Mark Spivak. 2012. Functional MRI in awake unrestrained dogs. PLoS ONE 7, 5. https://doi.org/10.1371/journal.pone.0038027

[2] Gregory S. Berns, Andrew M. Brooks, Mark Spivak, Kerinne Levy, and F. Range. 2017. Functional MRI in Awake Dogs Predicts Suitability for Assistance Work. Scientific Reports 7: 43704. https://doi.org/10.1038/srep43704

[3] John W S Bradshaw, Anne J. Pullen, and Nicola J. Rooney. 2015. Why do adult dogs "play"? Behavioural Processes 110 : 82-87. https://doi.org/10.1016/j.beproc.2014.09.023

[4] Stanley Coren. 2006. The intelligence of dogs: a guide to the thoughts, emotions, and inner lives or our canine companions. Free Press. Retrieved April $\quad 13, \quad 2017 \quad$ from https://books.google.com/books?id=nBzuXFdFECEC\&dq=the+intelligence + of + dogs\&lr=\&source=gbs_navlinks_s

[5] Stanley Coren. 2014. Do Puppy Personality Tests Predict Adult Dog Behaviors? Psychology Today.

[6] Deborah L. Duffy and James A. Serpell. 2012. Predictive validity of a method for evaluating temperament in young guide and service dogs. Applied Animal Behaviour Science 138, 99-109. https://doi.org/10.1016/j.applanim.2012.02.011

[7] E. Fonberg, E. Kostarczyk, and J. Prechtl. Training of instrumental responses in dogs socially reinforced by humans. The Pavlovian journal of biological science : official journal of the Pavlovian 16, 4: 183-193. https://doi.org/10.1007/BF03003358

[8] Jamie L Fratkin, David L Sinn, Erika A Patall, Samuel D Gosling, and Anja Widdig. 2013. Personality Consistency in Dogs: A MetaAnalysis. PLoS ONE 8, 1. https://doi.org/10.1371/

[9] Thomas Fuchs, Claude Gaillard, Sabine Gebhardt-Henrich, Silvia Ruefenacht, and Andreas Steiger. 2005. External factors and reproducibility of the behaviour test in German shepherd dogs in Switzerland. Applied Animal Behaviour Science 94: 287-301. https://doi.org/10.1016/j.applanim.2005.02.016

[10] Yuying Hsu and James A. Serpell. 2003. Development and validation of a questionnaire for measuring behavior and temperament traits in pet dogs. Journal of the American Veterinary Medical Association 223, 9: 1293-1300. https://doi.org/10.2460/javma.2003.223.1293

[11] Melody M. Jackson, Giancarlo Valentin, Larry Freil, et al. 2015. FIDO-Facilitating interactions for dogs with occupations: wearable communication interfaces for working dogs. Personal and Ubiquitous Computing 19, 1: 155-173. https://doi.org/10.1007/s00779-0140817-9

[12] Melody Moore Jackson, Yash Kshirsagar, Thad Starner, et al. 2013. FIDO - facilitating interactions for dogs with occupations. In Proceedings of the 17th annual international symposium on International symposium on wearable computers - ISWC '13, 81. https://doi.org/10.1145/2493988.2494334

[13] Niels Landwehr, Mark Hall, and Eibe Frank. 2005. Logistic model trees. Machine Learning 59, 1-2: 161-205. https://doi.org/10.1007/s10994-005-0466-3

[14] D R Lane, J Mcnicholas, and G M Collis. 1998. Dogs for the disabled: benefits to recipients and welfare of the dog. Applied Animal Behaviour Science 59: 49-60. Retrieved April 13, 2017 from http://ac.els-cdn.com/S0168159198001208/1-s2.0-S0168159198001208main.pdf?_tid=ceacacb2-207b-11e7-9a1e-00000aab0f01\&acdnat=1492110357_599c2e63ea740ca23965e88e936d1263

Proceedings of the ACM on Interactive, Mobile, Wearable and Ubiquitous Technologies, Vol. 1, No. 4, Article 127. Publication date: 
[15] Julie A Murphy. 1998. Describing categories of temperament in potential guide dogs for the blind. Applied Animal Behaviour Science 58: $163-178$.

[16] Willem J Netto and Doreen J U Planta. 1997. Behavioural testing for aggression in the domestic dog. Applied Animal Behaviour Science Plants/Applied Animal Behaviour Science 52, 52: 243-263.

[17] Anne Jennifer Pullen. 2011. Behavioural Indicators of Candidate Enrichment for Kennel-Housed Dogs.

[18] Nicola J. Rooney. 1999. Play behaviour of the domestic dog Canis familiaris and its effects on the dog-human relationship.

[19] Nicola J Rooney and John W S Bradshaw. 2003. Links between play and dominance and attachment dimensions of dog-human relationships. fournal of applied animal welfare science: FAAWS 6, 2: 67-94. https://doi.org/10.1207/S15327604JAWS0602_01

[20] G. Valentin, J. Alcaidinho, M. Jackson, A. Howard, and T. Starner. 2016. Creating Collar-sensed Motion Gestures for Dog-Human Communication in Service Applications. In Proceedings of the 2016 ACM International Symposium on Wearable Computers (ISWC '16).

[21] W. Volhard, J., \& Volhard. 1997. The canine good citizen: Every dog can be one. Howell Book House. Retrieved from http://www.volhard.com/pages/pat.php

[22] Deborah L. Wells. 2009. The Effects of Animals on Human Health and Well-Being. fournal of Social Issues 65, 3: 523-543. https://doi.org/10.1111/j.1540-4560.2009.01612.x

[23] International Association of Assistance Dog Partners. Retrieved April 13, 2017 from http://iaadp.org/

[24] Canine Companions for Independence - FAQs. Retrieved April 13, 2017 from http://www.cci.org/about/faqs.html\#question29

[25] Frequently Asked Questions about Service Animals and the ADA. Retrieved April 13, 2017 from https://www.ada.gov/regs2010/service_animal_qa.html

[26] 2012. U.S. Pet (Dog and Cat) Population Fact Sheet. Retrieved April 13, 2017 from http://www.bradfordlicensing.com/documents/petsfact-sheet.pdf

[27] 2015. Canine Companions for Independence 2015 Annual Report. Retrieved April 14, 2017 from http://www.cci.org/assets/files/2015annual-report.PDF

Received May 2015; revised August 2017; accepted October 2017 